ARTICLE

Received 9 Aug 2016 | Accepted 12 Dec 2016 | Published 1 Feb 2017

\title{
Ellipsoidal analysis of coordination polyhedra
}

\author{
James Cumby ${ }^{1} \&$ J. Paul Attfield ${ }^{1}$
}

The idea of the coordination polyhedron is essential to understanding chemical structure. Simple polyhedra in crystalline compounds are often deformed due to structural complexity or electronic instabilities so distortion analysis methods are useful. Here we demonstrate that analysis of the minimum bounding ellipsoid of a coordination polyhedron provides a general method for studying distortion, yielding parameters that are sensitive to various orders in metal oxide examples. Ellipsoidal analysis leads to discovery of a general switching of polyhedral distortions at symmetry-disallowed transitions in perovskites that may evidence underlying coordination bistability, and reveals a weak off-centre ' $d^{5}$ effect' for Fe ${ }^{3+}$ ions that could be exploited in multiferroics. Separating electronic distortions from intrinsic deformations within the low temperature superstructure of magnetite provides new insights into the charge and trimeron orders. Ellipsoidal analysis can be useful for exploring local structure in many materials such as coordination complexes and frameworks, organometallics and organic molecules.

\footnotetext{
${ }^{1}$ Centre for Science at Extreme Conditions and School of Chemistry, University of Edinburgh, West Mains Road, Edinburgh EH9 3JZ, UK. Correspondence and requests for materials should be addressed to J.P.A. (email: j.p.attfield@ed.ac.uk).
} 
S ymmetry group analysis provides a rigorous approach for quantifying distortions of coordination polyhedra but large numbers of modes are often present, for example, an $\mathrm{MX}_{6}$ octahedron has 3 rotations and 15 distortion modes. Continuous symmetry and shape distortion approaches provide some simplifications by parameterizing distortions as a numerical deviation from an idealized polyhedron, and by describing distorted structures in terms of similarity to more ideal polyhedral shapes $^{1-5}$. However, all of these methods require an appropriate reference polyhedron which may be difficult to define for irregular polyhedra such as 5- or 7-coordinate complexes. As a much more simple approach, single parameter approximations to describe polyhedra and their distortions are useful, but commonly used functions based on M-X distances $d$ such as $\Delta=\sigma^{2}(d) /$ $<d^{2}>$ (where variance $\sigma^{2}(d)=<d^{2}>-<d>^{2}$ and $<>$ denotes an average) are not sensitive to angular distortions, while angular variance functions ignore distance variations ${ }^{6-8}$.

A simple and general method for analysing distortions of polyhedra that takes account of shape irrespective of coordination number and geometry is to use an ellipsoidal approximation. Vertices of high symmetry polyhedra lie on the surface of a sphere. Deformations of the polyhedra transform the enclosing sphere into an ellipsoid and in this study we calculate the minimum bounding ellipsoid (MBE) of a given coordination polyhedron, which is the ellipsoid of smallest volume that encompasses all atoms, as described in 'Methods' section. The MBE can be calculated for any polyhedron, irrespective of coordination number and geometry. All geometric information for the polyhedron is reduced to the properties of the MBE, plus the displacement vector of the central atom relative to the ellipsoid centre for complexes lacking inversion symmetry. The principal ellipsoid radii are ordered $R_{1} \geq R_{2} \geq R_{3}$. Their mean $\langle R\rangle$ is related to the polyhedron size and their variance $\sigma^{2}(R)$ or s.d. $\sigma(R)=\sqrt{ } \sigma^{2}(R)$ quantifies polyhedral distortion. Ellipsoid shape is represented by the parameter $S=R_{3} / R_{2}-R_{2} / R_{1}$ which has range $-1 \leq S \leq 1$ where oblate (axially compressed) and prolate (axially stretched) ellipsoids respectively have $S<0$ and $S>0$, and a sphere has $S=0$. This method allows the magnitude of distortions of different symmetry to be compared directly, as shown in Fig. 1 for tetragonally and trigonally distorted octahedra. Our ellipsoidal approach has not been elaborated previously, although a best fit ellipsoid method was suggested for defining the centre of irregular polyhedra ${ }^{9,10}$, and an $\mathrm{MBE}$ method was recently used to characterize thermal expansion in perovskite-type $\mathrm{ABX}_{3}$ formates ${ }^{11}$.

Taking published crystal structures of metal oxides as examples, we demonstrate below that ellipsoidal analysis based on fitting the MBE provides new insights into phase transitions in perovskites, and complex distortions and electronic orders in iron oxides. Our approach has the advantages of providing sensitivity to both distance and angle distortions and of enabling straightforward comparison of coordination polyhedra of any size and symmetry with many fewer parameters than full symmetry mode analysis.

\section{Results}

Critical variations at perovskite tilt transitions. Perovskites are an important class of materials with diverse uses including ferroelectrics, for example, $\mathrm{BaTiO}_{3}$, magnetics and spintronics, for example, $\mathrm{La}_{1-x} \mathrm{Sr}_{x} \mathrm{MnO}_{3}$, and photovoltaics, for example, $\left(\mathrm{CH}_{3} \mathrm{NH}_{3}\right) \mathrm{PbI}_{3}$ (ref. 12). The ideal $\mathrm{AMX}_{3}$ perovskite structure is cubic with cuboctahedral $\mathrm{AX}_{12}$ and octahedral $\mathrm{BX}_{6}$ coordinations for which the MBEs are spheres. An important structural aspect is the tendency of perovskites to form superstructures of long-range ordered tilts or rotations of the $\mathrm{MX}_{6}$ octahedra, and 15 simple tilt systems have been classified ${ }^{13-15}$. Most analyses use structural quantities related to the octahedral tilt angle $(\phi)$ as the order parameter for transitions between these phases.

$\mathrm{LaAlO}_{3}$ has one of the simplest and most studied perovskite transitions, between low temperature rhombohedral (space group $R \overline{3} c$ ) and high temperature cubic $(P m \overline{3} m)$ structures at $T_{\mathrm{c}}=820 \mathrm{~K}$. The $R \overline{3} \mathrm{c}$ structure is formed by introduction of a single tilt with loss of some symmetry operations of the cubic phase and the transition is continuous (second order) ${ }^{16,17}$. Ellipsoidal analysis of the $\mathrm{LaO}_{12}$ and $\mathrm{AlO}_{6}$ polyhedra in $\mathrm{LaAlO}_{3}$ using published structural data ${ }^{17}$ shows that both ellipsoids change from spherical in the cubic phase to oblate in the $R \overline{3} c$ regime. Thermal variations of the ellipsoid radii and their average $\langle R>$ and s.d. $\sigma(R)$ are shown in Fig. 2.

Tilting of $\mathrm{AlO}_{6}$ octahedra deforms the $\mathrm{LaO}_{12}$ polyhedron and $\sigma(R)_{\mathrm{La}}$ is found to reproduce the temperature variation of the $\mathrm{AlO}_{6}$ tilt angle $\phi$, which is proportional to $t^{1 / 2}$ in the critical region approaching $T_{c}$ (where $t$ is the reduced temperature $\left.t=\left(T_{\mathrm{c}}-T\right) / T_{\mathrm{c}}\right)$ as shown in Fig. 2a. $\sigma(R)$ values for the $\mathrm{Al}$ ellipsoid are an order of magnitude smaller but they provide a sensitive measure of $\mathrm{AlO}_{6}$ octahedral strain. This is predicted to vary with $\phi^{2}$ and hence show a $\sim t^{1}$ critical variation ${ }^{18}$, as is observed in Fig. 2b. Hence ellipsoidal analysis provides a convenient and automatic way of accurately quantifying of the tilt and distortion of the $\mathrm{AlO}_{6}$ octahedra in $\mathrm{LaAlO}_{3}$, through $\sigma(R)_{\mathrm{La}}$ and $\sigma(R)_{\mathrm{Al}}$, respectively.

Symmetry-disallowed transitions in perovskites. Although several branches of continuous symmetry descent from the cubic $P m \overline{3} m$ structure are possible through successive tilting transitions, many investigated perovskites show symmetry-disallowed transitions. These have no group-subgroup relation between structures and hence are necessarily first order. Symmetrydisallowed transitions are relatively common in oxide perovskites, as shown in Supplementary Table 1, but the reason for their prevalence is unclear.

We have performed ellipsoidal analyses for many $\mathrm{ABO}_{3}$ perovskites where good quality structural data are available over multiple temperatures proximate to phase transitions. We use the ellipsoid shape parameter which has range $-1 \leq S \leq 1$, where negative and positive values respectively correspond to oblate and prolate distortions of $S=0$ spherical coordination. Symmetrydisallowed transitions between perovskite tilt superstructures are discovered to always change the sign of the shape parameter $(s(S)=+$ or -$)$ for at least one of the two coordination ellipsoids (Fig. 3), whereas this is sometimes but not always observed for symmetry-allowed transitions. Prominent examples are below with further information in Supplementary Note 1 and data citations in Supplementary Table 1.

Orthorhombic-rhombohedral transitions are observed in many perovskites. $\mathrm{RAlO}_{3}$ perovskites $(\mathrm{R}=\mathrm{La}, \mathrm{Ce}, \mathrm{Pr}$ and $\mathrm{Nd})$ all display the $R \overline{3} c \rightarrow P m \overline{3} m$ transition described above for $\mathrm{LaAlO}_{3}$ (Fig. 3a,b and Supplementary Fig. 1). At lower temperatures, $\mathrm{CeAlO}_{3}$ and $\mathrm{PrAlO}_{3}$ also show a symmetry-disallowed orthorhombic Imma $\rightarrow$ rhombohedral $R \overline{3} c$ transition at which the shapes of both $\mathrm{R}$ and $\mathrm{Al}$ ellipsoids change, from prolate $(S>0)$ in the Imma structure to oblate $(S<0)$ in the $R \overline{3} c$ regime, as shown in Fig. 3a,b. Other $\mathrm{ABO}_{3}$ perovskites having this Imma $\rightarrow R \overline{3} c\left(\mathrm{BaCeO}_{3}\right)$ or a Pnma $\rightarrow R \overline{3} c\left(\mathrm{PrNiO}_{3}, \mathrm{LaCrO}_{3}\right.$ and $\mathrm{LaGaO}_{3}$ ) symmetry-disallowed transition also display a sign change in at least one of the two ellipsoids as shown in Fig. $3 \mathrm{c}$ and Supplementary Table 1.

Symmetry-disallowed orthorhombic-tetragonal transitions are observed at high temperatures in a few perovskite materials. The Imma $\rightarrow \mathrm{I} 4 / \mathrm{mcm}$ transitions in $\mathrm{BaPbO}_{3}$ and $\mathrm{BaTbO}_{3}$ and the 

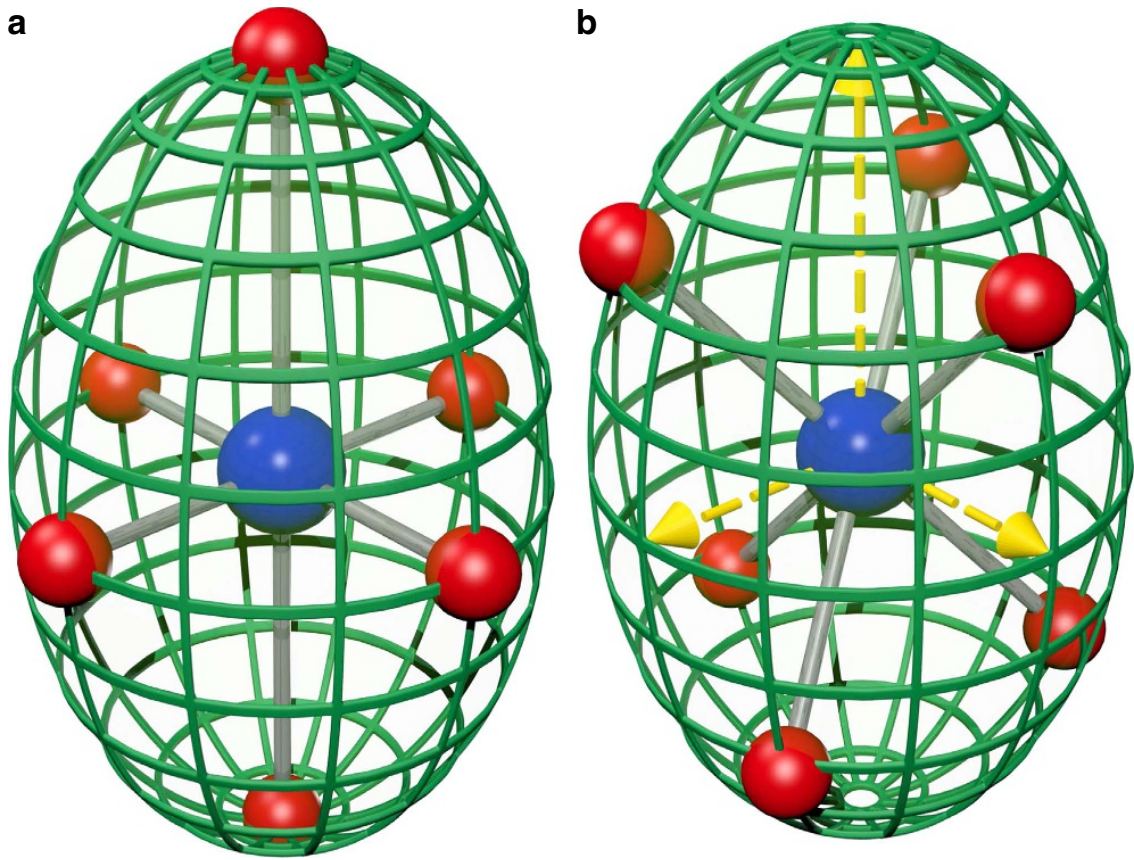

Figure 1 | Ellipsoidal fits to distorted octahedra. (a) The fitted $M B E$ (green net) for a tetragonally elongated $M X_{6}$ octahedron $(M / X=b l u e / r e d ~ s p h e r e s)$ where $M-X$ bond distances differ but cis $X-M-X$ angles are $90^{\circ}$. (b) $M B E$ fit to a trigonally distorted $M X_{6}$ octahedron, where $M-X$ distances are equal but cis $\mathrm{X}-\mathrm{M}-\mathrm{X}$ angles deviate from $90^{\circ}$. The two distorted octahedra have identical prolate MBEs with principal ellipsoid radii shown as yellow arrows in $\mathbf{b}$.
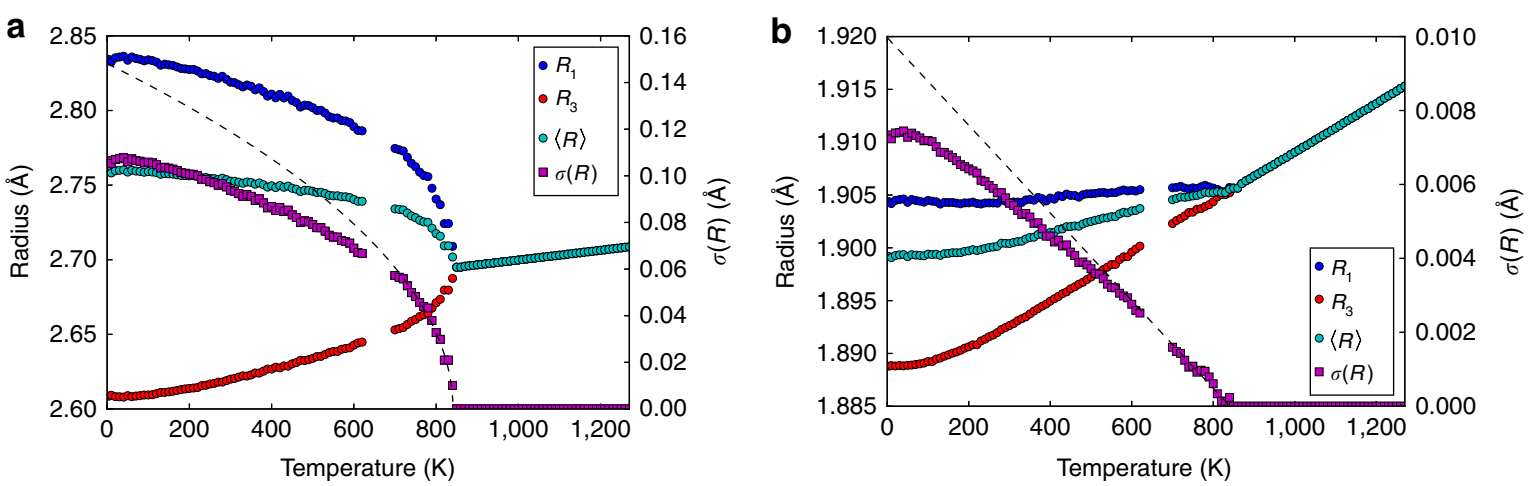

Figure 2 | Ellipsoidal analysis of the $\mathbf{R} \overline{\mathbf{3}} \mathbf{c} \rightarrow \mathbf{P} \mathbf{m} \overline{\mathbf{3}} \mathbf{m}$ structural transition in LaAlO $\mathbf{3}_{\text {. }}$ (a) Variations of the ellipsoid radii, their average $<R>$ and s.d. $\sigma(R)$ for the $\mathrm{LaO}_{12}$ polyhedron, with a $t^{1 / 2}$ fit to $\sigma(R)$ points above $700 \mathrm{~K}$ shown. (b) Variations of the ellipsoid radii, $\left\langle R>\right.$ and $\sigma(R)$ for the $\mathrm{AlO}_{6}$ octahedron showing linear $\sigma(R) \sim t$ behaviour approaching the transition. $t$ is the reduced temperature $t=\left(T_{\mathrm{c}}-T\right) / T_{\mathrm{c}}$.

Pnma $\rightarrow \mathrm{I} 4 / \mathrm{mcm}$ transition in $\mathrm{CaTiO}_{3}$ all show a change of $s(S)_{\mathrm{B}}$ from positive to negative (Fig. $3 \mathrm{~d}$ ).

The famous perovskite $\mathrm{BaTiO}_{3}$ provides a striking example of $s(S)$ changes. This material forms a series of ferroelectric phases where changes in the polarization defined by off-centre Ti displacement directions $[111] \rightarrow[110] \rightarrow[100] \rightarrow[000]$ (nonpolar) gives rise to the $R 3 m \rightarrow A m m 2 \rightarrow P 4 m m \rightarrow P m \overline{3} m$ sequence of phase transitions ${ }^{19,20}$. The first two transitions are symmetry-disallowed although in principle the magnitude of the polarization can vary continuously through them. Evolution of the off-centre Ti displacements and their local order through the transitions have been studied extensively ${ }^{21,22}$, but accompanying distortions of the $\mathrm{TiO}_{6}$ octahedron are less explored. Shape parameters $S_{\mathrm{Ba}}$ and $S_{\mathrm{Ti}}$ calculated from published structural data $^{23,24}$ both show a striking switching behaviour through the $R 3 m \rightarrow A m m 2 \rightarrow P 4 m m$ transitions in Fig. 3e,f. In contrast, the magnitudes of the off-centre displacements of the cations vary continuously through the transitions. Hence, the ellipsoidal shape parameters provide complementary information to the off-centre distortions. The former demonstrate abrupt changes in local coordination consistent with the first order transitions and further illustrate the general observation of sign switching at symmetry-disallowed changes, while the latter illustrate how the polarization magnitude effectively acts as a primary order parameter for the entire sequence of transitions.

The sign changes in ellipsoidal shape parameter at symmetrydisallowed perovskite phase transitions provide an important structural insight that has not been previously reported, even for much-studied materials like $\mathrm{BaTiO}_{3}$. The prevalence of sign changes suggests that there is a general bistability to the coordination polyhedra in tilted perovskite superstructures. In the simplest ellipsoidal approximation the distortions can be either prolate or oblate, and if these two states are of similar energy then changes of temperature or other tuning variables such as pressure or composition can lead to changes between them. Prolate and oblate distortions are generally accommodated by different tilt systems, resulting in frequent observation of symmetry-disallowed transitions in perovskites. The switching may be enhanced 

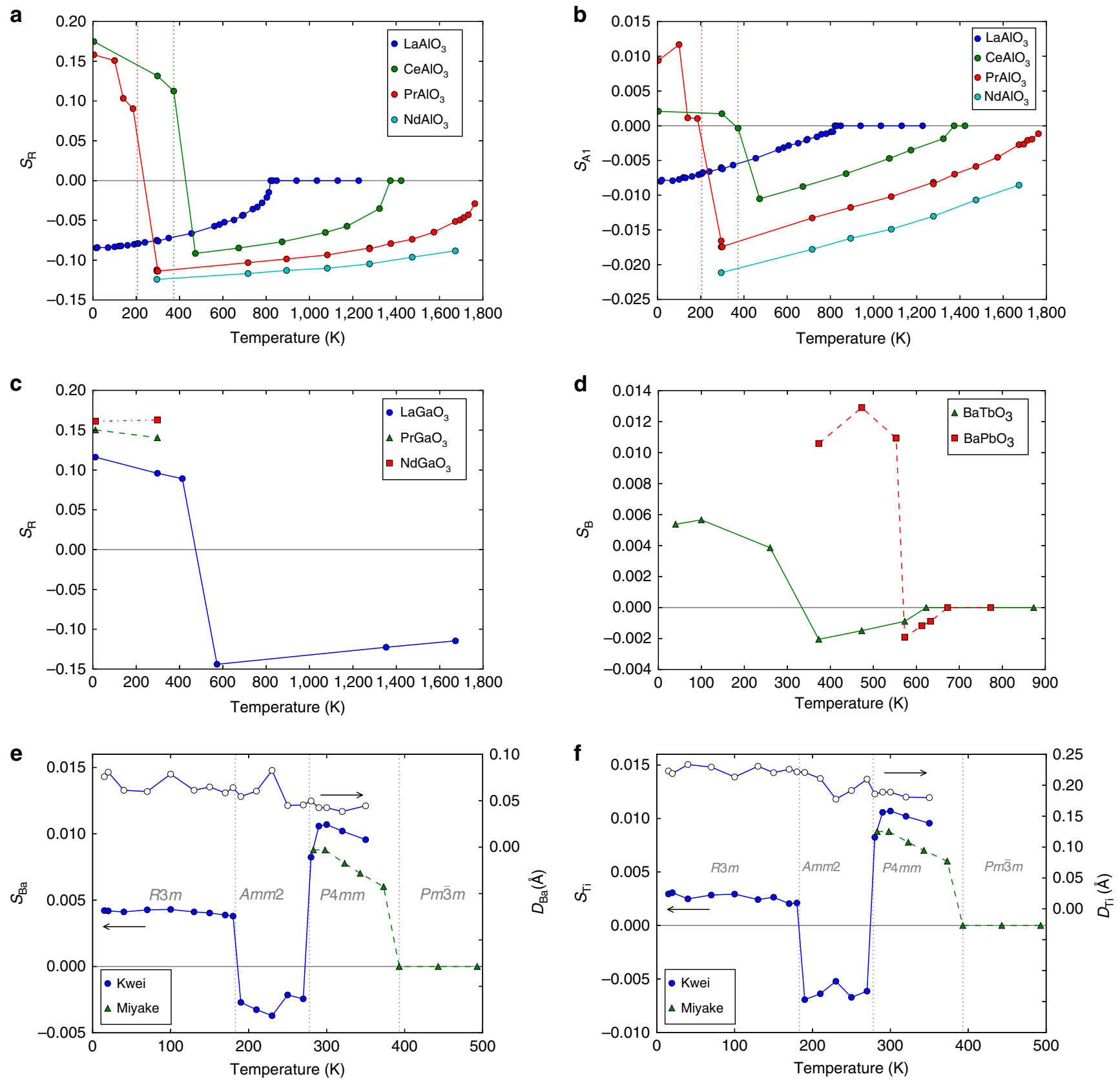

Figure 3 | Thermal variations of the ellipsoidal shape parameter $\mathbf{S}$ for $\mathbf{A B O}_{\mathbf{3}}$ perovskites. Changes of sign in $\mathrm{S}$ are observed at the symmetry-disallowed transitions. (a) $S_{R}$ and (b) $S_{A l}$ shape parameters for $R_{A l O}$ perovskites $(R=L a, C e$, $\mathrm{Pr}$ and $\mathrm{Nd}$ ) with sign changes at the $I m m a \rightarrow R \overline{3} \mathrm{c}$ transition (denoted by vertical lines). (c) $S_{R}$ variation in $\mathrm{RGaO}_{3}$ perovskites showing a sign change at the $P n m a \rightarrow R \overline{3} c$ transition for $\mathrm{LaGaO}_{3}$. (d) $\mathrm{S}_{\mathrm{B}}$ versus temperature for $\mathrm{BaPbO}{ }_{3}$ and $\mathrm{BaTbO}_{3}$ through their $1 \mathrm{mma} \rightarrow 14 / \mathrm{mcm}$ transitions at 573 and $280 \mathrm{~K}$ respectively. (e) $\mathrm{S}_{\mathrm{Ba}}$ and (f) $S_{\mathrm{Ti}}$ variations through the three structural phase transitions of $\mathrm{BaTiO}_{3}$ using published structural data from Kwei et al. ${ }^{23}$ and Miyake et al. ${ }^{24}$ Off-centre cation displacements $D$ calculated from the former data are also shown.

by electronic instabilities such as weak crystal field effects for $\mathrm{Ce}^{3+}$ and $\mathrm{Pr}^{3+}$ in $\mathrm{RAlO}_{3}$ or the second order Jahn-Teller instability of $\mathrm{Ti}^{4+}$ in $\mathrm{BaTiO}_{3}$.

Iron oxide polyhedra. Ellipsoidal analysis provides a useful approximation for comparing the distortions and shapes of polyhedra with different coordination numbers and geometries through data-mining of crystallographic databases such as Inorganic Crystal Structure Database (ICSD) or Cambridge Structural Database (CSD) (refs 25,26). These aspects are illustrated by using 499 iron oxide polyhedra from 390 structures in the ICSD where $98 \mathrm{Fe}^{2+}$ and $401 \mathrm{Fe}^{3+}$ sites have coordination numbers between 4 and 6 . Results are shown in Fig. 4 and Supplementary Fig. 2, and further information is in Supplementary Note 2 .

The plot of ellipsoid s.d. $\sigma(R)$ versus mean radius $<R>$ plot in Fig. 4a shows differing features for the common $\mathrm{FeO}_{n}$ coordinations. The distributions for $\mathrm{FeO}_{4}$ tetrahedra and $\mathrm{FeO}_{6}$ octahedra both have many points near $\sigma(R)=0$, reflecting their high ideal symmetries, and are clustered around $\langle R\rangle \approx d$ where $d$ is an average $\mathrm{Fe}-\mathrm{O}$ distance for each coordination. Ideal values based on standard ionic radii for the predominant high spin $\mathrm{Fe}^{3+}$ state in tetrahedral and octahedral geometries are $d=1.89$ and $2.05 \AA$, 
a

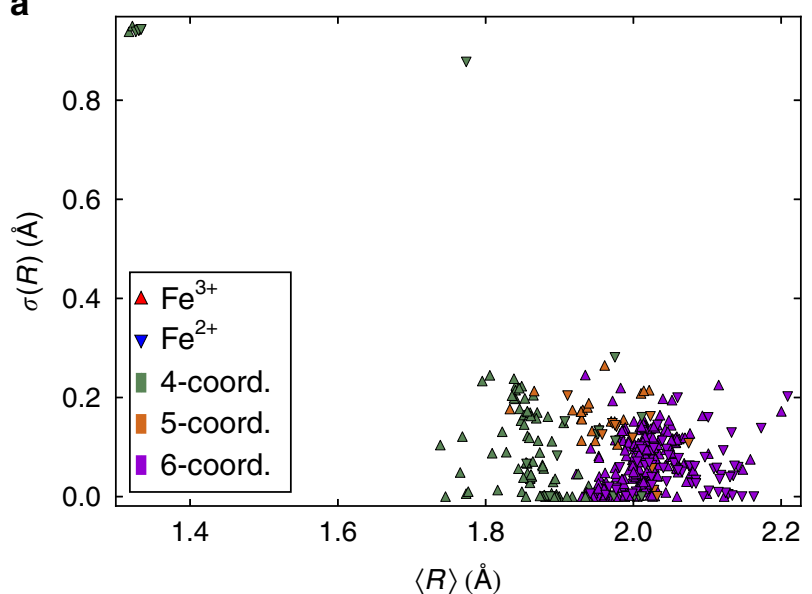

b

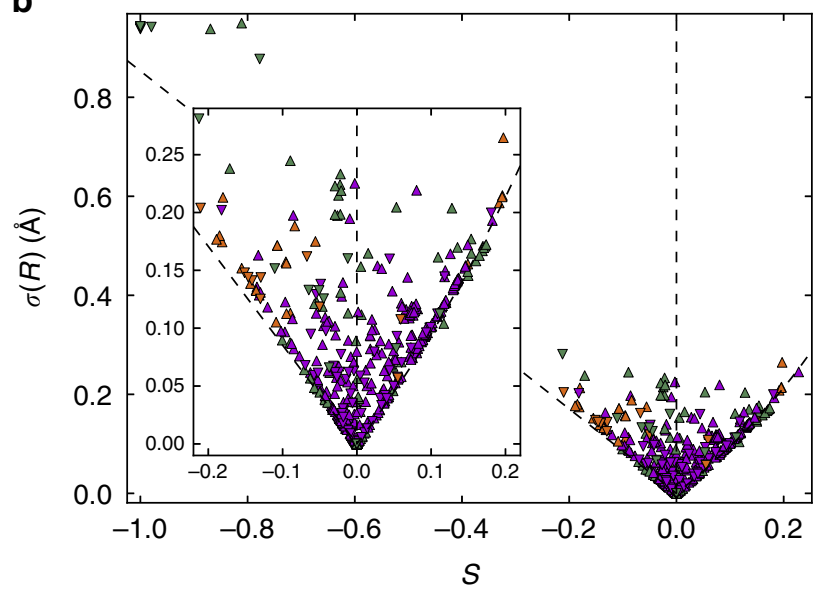

Figure 4 | Ellipsoidal parameter plots for literature $\mathbf{F e O}_{\boldsymbol{n}}$ polyhedra. $499 \mathrm{FeO}_{n}$ polyhedra in iron oxides ( 98 for $\mathrm{Fe}^{2+}$ and 401 for $\mathrm{Fe}^{3+}$ ) taken from the ICSD with Fe coordination numbers $n$ between 4 and 6 are shown. (a) s.d. $\sigma(R)$ versus mean ellipsoid radius $\langle R\rangle$. (b) $\sigma(R)$ versus shape parameter $S$ showing limits for oblate $(S<0)$, geometrically scalene $(S=0)$ and prolate $(S>0)$ ellipsoids taking $R_{2}=1.81 \AA$. The inset (which does not obscure any points on the main plot) shows an expansion for the region near $S=0$.

respectively. Five-coordinate polyhedra are rarer and have less regular geometries, as evidenced by the bulk of the distribution lying between $\sigma(R) \approx 0.1$ and $0.3 \AA$. Highly anisotropic polyhedra have $\langle R>$ values far from $d$ and large values of $\sigma(R)$. Ellipsoids describing $\mathrm{FeO}_{4}$ square planes have principal axes $R_{1} \approx R_{2} \approx d$ and $R_{3} \approx 0$ so $<R>\approx(2 / 3) d$ and $\sigma(R) \approx(\sqrt{ } 2 / 3) d$. The points at $\sigma(R)>0.8 \AA$ show this coordination, with a cluster of flat square planes at $\langle R>\approx 1.3 \AA$ and a more distorted variant at $<R>=1.8 \AA$.

The plot of $\sigma(R)$ versus ellipsoid shape $S$ in Fig. $4 \mathrm{~b}$ is another useful way to observe the spectrum of iron oxide polyhedra. $S$ varies from near -1 for square planar complexes in the oblate distortion limit through many distorted and regular tetrahedra and octahedra at $S \approx-0.2$ to 0.3 . The V-shaped appearance of the distribution results from the most symmetric ellipsoid shapes imposing lower limits on $\sigma(R)$; oblate coordinations $(S<0)$ have $\sigma(R)=-(\sqrt{ } 2 / 3) R_{2} S$ while prolate ellipsoids $(S>0)$ have $\sigma(R)=(\sqrt{ } 2 / 3) R_{2} S /(1-S)$. These limits are shown on the plot together with the line for geometrically scalene (triaxial) ellipsoids, which have $S=0$ but are non-spherical with $R_{3} / R_{2}=R_{2} / R_{1}$ but $R_{1} \neq R_{2} \neq R_{3}$. This distortion is seen to be rather rare compared with oblate and prolate coordinations.

Electronic orders in magnetite. Analysis of the coordination environment is important when subtle electronic features such as charge or orbital orders are present. A famous example is the iron oxide spinel magnetite, $\mathrm{Fe}_{3} \mathrm{O}_{4}$, where proposed $\mathrm{Fe}^{2+} / \mathrm{Fe}^{3+}$ charge order below the Verwey transition at $125 \mathrm{~K}$ has been controversial for many decades ${ }^{27-35}$. Conventional analysis of specific radial and tetragonal Jahn-Teller modes in the refined superstructure was recently used to demonstrate $\mathrm{Fe}^{2+} / \mathrm{Fe}^{3+}$ charge order and $\mathrm{Fe}^{2+}$ orbital order over 16 independent $\mathrm{B}$-site $\mathrm{FeO}_{6}$ octahedra ${ }^{33}$. Short distances from $\mathrm{Fe}^{2+}$ sites to $\mathrm{B}$-site neighbours further revealed weak direct $\mathrm{Fe}-\mathrm{Fe}$ bonding effects that give rise to linear $\mathrm{Fe}_{3}$ units known as trimerons. Comparison of ellipsoidal parameter distributions for the $16 \mathrm{FeO}_{6}$ octahedra in magnetite, which are constrained through connectivity and cooperative electronic distortions, against the large unconstrained set of six-coordinate iron oxide polyhedra from the above analysis of literature structures in Fig. 5 provides useful structural insights.

The s.d. $\sigma(R)$ versus mean ellipsoid radius $\langle R>$ plot for the literature $\mathrm{FeO}_{6}$ polyhedra in Fig. 5a has little structure other than a small displacement between the $\langle R\rangle$ histograms (which display the number $N$ of polyhedra within each interval as $\log (N+1)$ to observe variations from $N=0$ to $N>100)$ for $\mathrm{Fe}^{2+}$ and $\mathrm{Fe}^{3+}$ according to their ideal $\mathrm{Fe}-\mathrm{O}$ distances based on ionic radii which are $d=2.18$ and $2.05 \AA$. However, the corresponding plot for magnetite (Fig. 5b) has a V-shape with the smallest $\mathrm{Fe}^{3+}$ and largest $\mathrm{Fe}^{2+}$ states having comparably large $\sigma(R)$ values. This reflects the highly correlated nature of the distortions in magnetite, as each octahedron shares edges with six neighbours leading to an increase in $\sigma(R)$ with increasing deviation in $\langle R\rangle$ from the overall structural average. However, instead of meeting with a symmetric V-shape, there is a significant excess in $\sigma(R)$ for the $\mathrm{Fe}^{2+}$ line relative to that for the $\mathrm{Fe}^{3+}$ line where the two distributions meet at $\langle R>=2.06 \AA$ (corresponding to the average octahedral $\mathrm{Fe}-\mathrm{O}$ bond length). This excess distortion provides direct evidence for the intrinsic Jahn-Teller distortion of octahedra containing triply-degenerate, high spin $3 \mathrm{~d}^{6} \mathrm{Fe}^{2+}$ whereas $3 d^{5} \mathrm{Fe}^{3+}$ is non-degenerate. The $0.03 \mathrm{~A}$ magnitude of the excess in $\sigma(R)$ is equivalent to a tetragonal compression where four coplanar $\mathrm{Fe}-\mathrm{O}$ bonds expand by $0.02 \AA$ and the other pair contract by $0.04 \AA$. Hence the ellipsoidal $\sigma(R)$ versus $<R>$ plot is valuable as it enables the single-ion electronic distortion due to Jahn-Teller instability for $\mathrm{Fe}^{2+}$ states to be separated from the correlated deformations of the octahedra due to their high connectivity in the magnetite structure. This is not easily done when using simpler measures of distortion such as the $\Delta$ parameter.

Magnitudes $D$ of the $\mathrm{Fe}$ atom displacement relative to the ellipsoid centre are plotted against mean ellipsoid radius $\langle R\rangle$ in Fig. 5c. Neglecting a few outliers, the distribution has a peak at $\langle R>\approx 2.0 \AA$ (close to the average $\mathrm{Fe}-\mathrm{O}$ distance for the predominant $\mathrm{Fe}^{3+}$ state) showing that many near-regular $\mathrm{FeO}_{6}$ polyhedra have off-centre instabilities. (Off-centre displacements might be expected to predominate in the most distorted octahedra, but the data do not support this expectation.) Octahedra at a crystallographic inversion centre have $D=0$ but there is a striking difference between the $D>0$ distributions for $\mathrm{Fe}^{2+}$ and $\mathrm{Fe}^{3+}$ states. $\mathrm{Fe}^{2+}$ displacements fall off with increasing $D$ values up to a maximum value of $0.19 \AA$, whereas $\mathrm{Fe}^{3+}$ displacements range up to $D=0.30 \AA$ with a peak in the distribution at $D \approx 0.15 \AA$. This disparity is greater than might be expected from crystal field effects (as $\sigma(R)$ distributions for $\mathrm{Fe}^{2+}$ and $\mathrm{Fe}^{3+}$ in Fig. 5a are quite similar) and implies that 
a

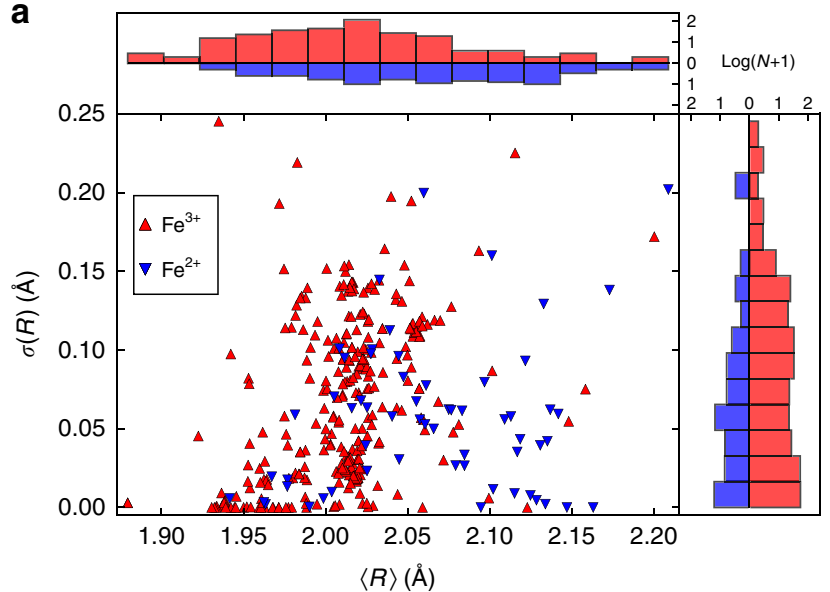

C

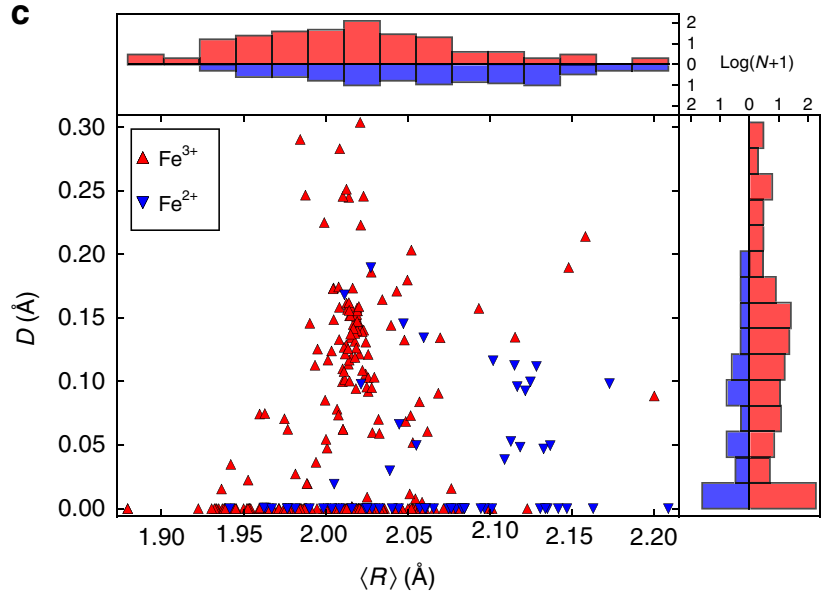

b

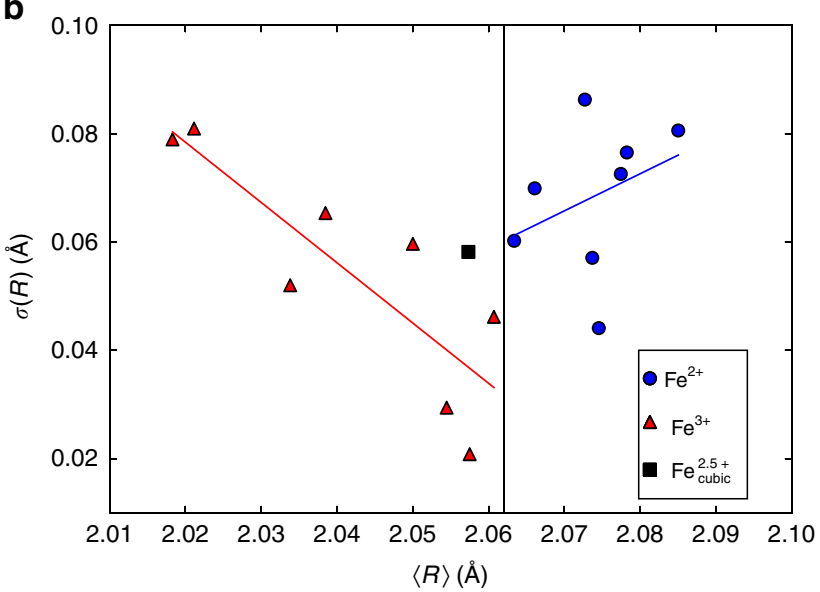

d

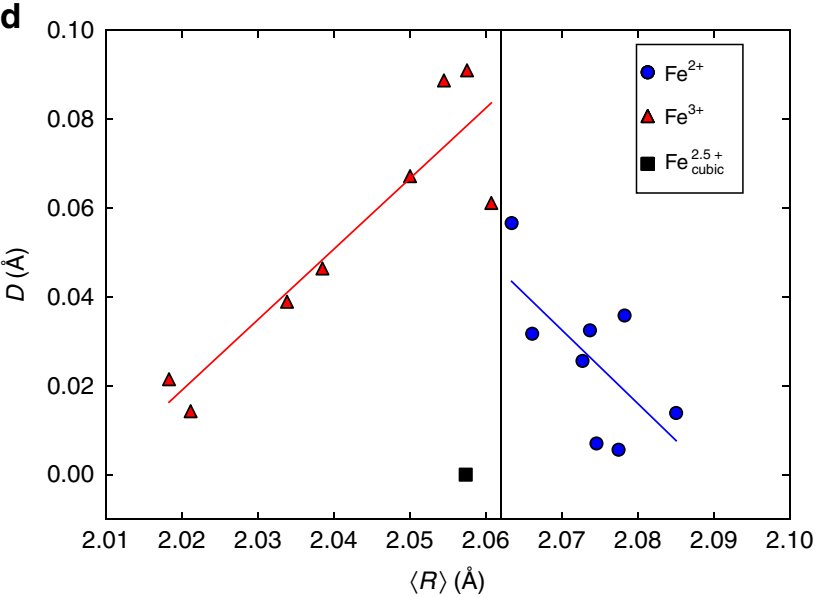

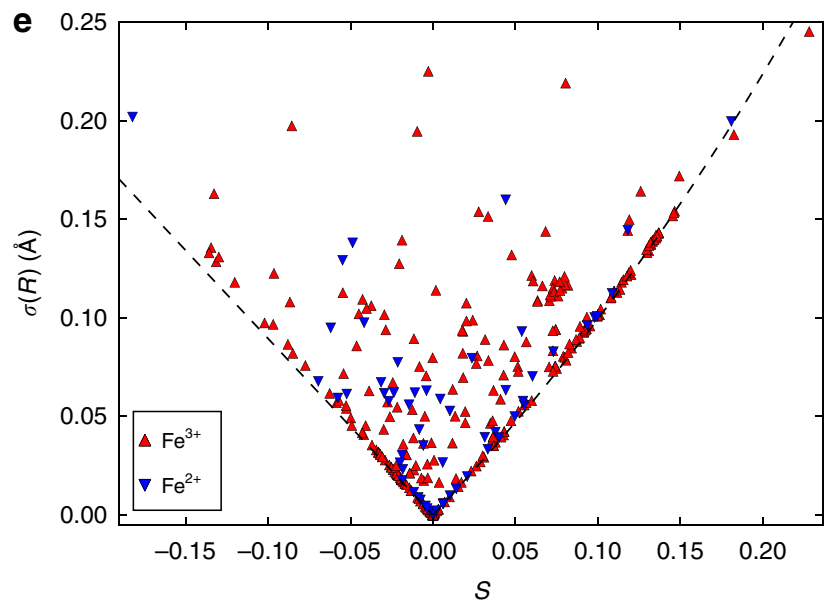

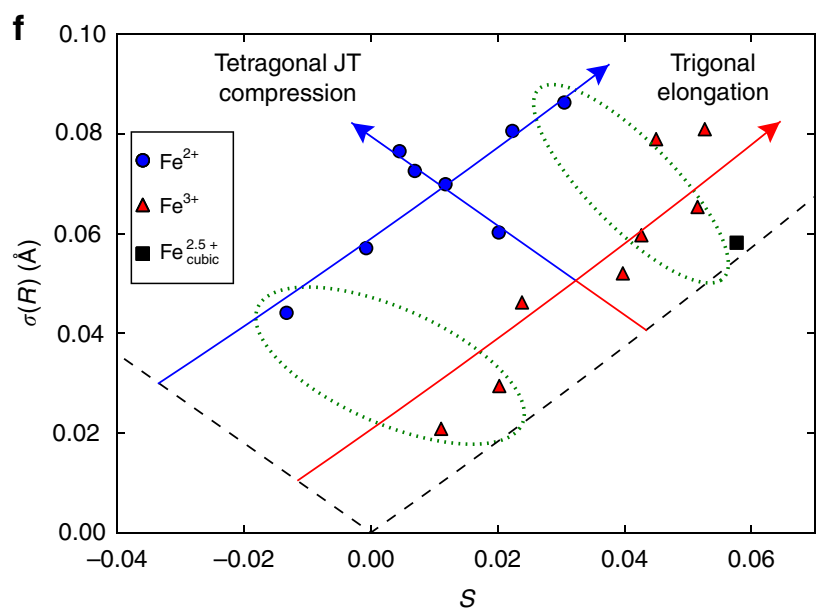

Figure 5 | Ellipsoidal parameters for $\mathbf{F e O}_{\mathbf{6}}$ polyhedra. The plots compare ellipsoidal parameters from iron oxide structures in the ICSD with those in magnetite. Histograms show distributions as $\log (\mathrm{N}+1)$ for $N$ polyhedra within each interval. Plots $(\mathbf{a}, \mathbf{c}, \mathbf{e})$ describe $359 \mathrm{FeO}_{6}$ polyhedra from 317 structures in ICSD. (b,d,f) are for the 16 independent $\mathrm{B}$-site $\mathrm{FeO}_{6}$ octahedra in the reported magnetite superstructure at $90 \mathrm{~K}$ (ref. 33), and the single average $\mathrm{Fe}^{2.5}+\mathrm{O}_{6}$ octahedral site in the high temperature cubic structure at $130 \mathrm{~K}$ is also shown ${ }^{28}$. (a) s.d. $\sigma(R)$ versus mean ellipsoid radius $<R>$ for ICSD FeO structures. (b) $\sigma(R)$ versus $<R>$ for $\mathrm{FeO}_{6}$ octahedra in magnetite. (c) Displacements $D$ of the cations from their ellipsoid centres versus $<R>$ for ICSD $\mathrm{FeO}_{6}$ structures. (d) $D$ versus $<R>$ for $\mathrm{FeO}_{6}$ octahedra in magnetite. (e) $\sigma(R)$ versus $\sigma(R)$ versus shape parameter $S$ showing limits for oblate $(S<0)$ and prolate $(S>0)$ ellipsoids for ICSD FeO ${ }_{6}$ structures taking $R_{2}=1.9 \AA$. (f) $\sigma(R)$ versus $S$ for $\mathrm{FeO}_{6}$ octahedra in magnetite, showing that points within the $\mathrm{Fe} \mathrm{e}^{2+}$ and $\mathrm{Fe}^{3+}$ distributions lie parallel to the prolate (trigonal elongation) and oblate (tetragonal Jahn-Teller compression) ellipsoid limits. Sets of three sites corresponding to the $\mathrm{Fe}^{3+}-\mathrm{Fe}^{2+}-\mathrm{Fe}^{3+}$ trimerons with the smallest and largest prolate distortions are encircled; assignments for all trimerons are shown in Supplementary Fig. 3. 
another influence is significant. This is likely a weak ' $d^{5}$ effect' for the half-filled $d$-shell of $\mathrm{Fe}^{3+}$, similar to the much stronger $d^{0}$ effect that gives rise to substantial off-centre displacements (second order Jahn-Teller distortions) for cations such as $\mathrm{Ti}^{4+}$ in $\mathrm{BaTiO}_{3}$ above. $d^{0}$ distortions have previously been analysed by continuous shape measures ${ }^{36}$. Recent calculations have suggested that off-centre displacements are favourable for $\mathrm{Fe}^{3+}$ in trigonal bipyramidal coordination ${ }^{37}$, and they are of interest for possible multiferroics where both electric and magnetic polarizations are generated by the $d^{5}$ configuration.

Linear variations of $D$ with $<R>$ are observed for both $\mathrm{Fe}^{2+}$ and $\mathrm{Fe}^{3+}$ states in low temperature magnetite in Fig. 5d, but with an excess displacement $\Delta D \approx 0.04 \AA$ for $\mathrm{Fe}^{3+}$ ions where the two distributions meet at $\langle R\rangle=2.06 \AA$. Previous structural analysis showed that these $\mathrm{Fe}^{3+}$ displacements are associated with trimeron formation ${ }^{33,35}$, as $\mathrm{Fe}^{3+}$ ions move towards the spatially ordered lobes of $t_{2 \mathrm{~g}}$ orbitals of adjacent $\mathrm{Fe}^{2+}$ states resulting in weak $\mathrm{Fe}-\mathrm{Fe}$ bonds. However, in light of the general analysis of $\mathrm{Fe}$ displacements revealed in Fig. $5 \mathrm{c}$, the weak ' $d^{5}$ effect' may also enhance the $\mathrm{Fe}^{3+}$ displacements and hence assist trimeron formation in magnetite.

The $\mathrm{V}$-shaped distribution in the plot of s.d. $\sigma(R)$ versus ellipsoid shape $S$ for literature $\mathrm{FeO}_{6}$ polyhedra in Fig. 5e has lower limits on $\sigma(R)$ corresponding to symmetric ellipsoid shapes as described above, and many polyhedra lie close to the oblate $(S<0)$ or prolate $(S>0)$ limits reflecting their high crystallographic symmetries. The corresponding plot for magnetite in Fig. 5f shows remarkable structure giving new insights into the electronic distortion. Points for the $16 \mathrm{FeO}_{6}$ octahedra fall close to three lines parallel to the symmetric ellipsoid limits. The spinel lattice imposes a prolate trigonal distortion on the unique $\mathrm{FeO}_{6}$ octahedron in the high temperature cubic structure (like that shown in Fig. 1b), and this is broadened into a dispersion of distortions close to the prolate limiting line for the $\mathrm{Fe}^{3+}$ type states in the low temperature structure. The $\mathrm{Fe}^{2+}$ distribution is shifted parallel to the oblate limit, as tetragonal Jahn-Teller compression of $\mathrm{Fe}^{2+} \mathrm{O}_{6}$ octahedra gives oblate ellipsoidal distortion. However the points for these octahedra are highly structured and lie very close to two lines, one at fixed oblate distortion, showing dispersed trigonal distortions at constant Jahn-Teller deformation, and the other at a constant average trigonal distortion with varying Jahn-Teller effect. Further analysis of this distribution is required but it appears to be related to trimeron formation, as the three sites in each unit have similar prolate distortions as shown for the least and most distorted trimerons on Fig. 5f, and for all trimerons on Supplementary Fig. 3. This structural correlation within the octahedra forming each trimeron was not evident in previous analyses using conventional symmetry group analysis ${ }^{33}$. Overall, the $\sigma(R)$ versus $S$ plot provides an elegant separation of the singleion electronic distortion, which is tetragonal and oblate, from the trigonal prolate deformations that are intrinsic to the distorted spinel lattice, with the two effects behaving as additive vectors parallel to the ellipsoid limits.

\section{Discussion}

The above results demonstrate the utility of ellipsoidal analysis of coordination polyhedra. Our ellipsoidal approximation based on fitting the MBE has the advantages of providing sensitivity to both distance and angle distortions and of enabling straightforward comparison of coordination polyhedra of any size and symmetry with many fewer parameters than full symmetry mode analysis. Although the derived ellipsoidal parameters are not predicated on any particular type of distortion being present, they are shown to be highly sensitive to the orders of interest in the metal oxide examples presented.

Ellipsoidal analysis provides a convenient parameterization of octahedral tilts and strains in perovskites and has led to discovery of a general switching of polyhedral distortions at symmetrydisallowed transitions that may evidence underlying coordination bistability. This method is also useful for comparing large numbers of structures from crystallographic databases, here providing evidence for a weak off-centre ' $d^{5}$ effect' for $\mathrm{Fe}^{3+}$ ions that may be exploited in multiferroics. Ellipsoidal plots for the magnetite superstructure have enabled local electronic distortions to be separated from correlated lattice deformations, providing new representations of the complex electronic order for future analysis.

Coordination polyhedra are ubiquitous to chemical structure so ellipsoidal analysis is likely to prove an equally useful tool for probing coordination complexes and frameworks, organometallics and organic molecules, and could even be applied to larger scale packings of molecules or nanoparticles.

\section{Methods}

MBE fitting. The MBE for a given coordination polyhedron is calculated using a Khachiyan minimization algorithm ${ }^{38}$, resulting in three principal radii and a rotation matrix for the ellipsoid, and off-centre displacement $D$ of the central atom. The principal ellipsoid radii are ordered $R_{1} \geq R_{2} \geq R_{3}$. Their mean $<R>$ is related to the polyhedron size and their variance $\sigma^{2}(R)$ or s.d. $\sigma(R)=\sqrt{ } \sigma^{2}(R)$ quantifies distortion. $S=R_{3} / R_{2}-R_{2} / R_{1}$ describes ellipsoid shape. Values of $\langle R\rangle$, $\sigma(R)$ and $S$ enable straightforward analysis and comparison of coordination polyhedra of any size and symmetry.

The MBE fitting algorithm is implemented within the PIEFACE (PolyhedraInscribing Ellipsoids For Analysing Crystallographic Environments) software package which uses Python 2.7. PIEFACE fits an ellipsoid to a set of points in Cartesian coordinates to within a supplied tolerance; if the points are coplanar then $R_{3}$ is set to zero. The algorithm can be accessed directly or operated via simple graphical or command-line interfaces which allows direct input of CIF files, with optional control over parameters such as which atoms are coordinating, the maximum radius to use for coordination searching, and the fit tolerance. A number of other routines have also been implemented for data analysis within the PIEFACE package including output of ellipsoid parameters to a number of formats and interactive visualization of the resulting ellipsoids.

Crystallographic data selection. Structural data were taken from published crystal structures. Where possible, structures obtained from neutron diffraction refinement were used to ensure accurate oxygen positions in the presence of heavy atoms. In the perovskite phase transition examples, variable temperature data were extracted from a single study wherever possible to avoid problems of systematic offsets between different experiments. For analysis of Fe oxide materials, polyhedra with Fe-coordinating atoms other than oxygen were rejected, as were mixed-cation sites with $\mathrm{Fe}$ occupations below $80 \%$. Only ambient pressure and temperature structures were used.

Code availability. Version 1.0.0 of PIEFACE used in this study is archived at doi:10.5281/zenodo.198370. Code updates are available at https:/github.com/jcumby/PIEFACE.

Data availability. All structural data used for ellipsoid fitting were taken from published crystal structures in the ICSD (version 2015.2) ${ }^{25}$, except for those for the variable temperature study of $\mathrm{LaAlO}_{3}$ shown in Fig. 2 which were taken from ref. 17 .

\section{References}

1. Pinsky, M. \& Avnir, D. Continuous symmetry measures. 5. The classical polyhedra. Inorg. Chem. 37, 5575-5582 (1998).

2. Alvarez, S., Avnir, D., Llunell, M. \& Pinsky, M. Continuous symmetry maps and shape classification. The case of six-coordinated metal compounds. New J Chem. 26, 996-1009 (2002).

3. Echeverria, J. \& Alvarez, S. Application of symmetry operation measures in structural inorganic chemistry. Inorg. Chem. 47, 10965-10970 (2008).

4. Alvarez, S., Menjon, B., Falceto, A., Casanova, D. \& Alemany, P. Stereochemistry of complexes with double and triple metal-ligand bonds: a continuous shape measures analysis. Inorg. Chem. 53, 12151-12163 (2014).

5. Alvarez, S. et al. Shape maps and polyhedral interconversion paths in transition metal chemistry. Coord. Chem. Rev. 249, 1693-1708 (2005). 
6. Alonso, J. A., Martinez-Lope, M. J., Casais, M. T. \& Fernandez-Diaz, M. T. Evolution of the jahn-teller distortion of $\mathrm{MnO}_{6}$ octahedra in $\mathrm{RMnO}_{3}$ perovskites $(\mathrm{R}=\mathrm{Pr}, \mathrm{Nd}, \mathrm{Dy}, \mathrm{Tb}, \mathrm{Ho}, \mathrm{Er}, \mathrm{Y})$ : a neutron diffraction study. Inorg. Chem. 39, 917-923 (2000).

7. Baur, W. H. The geometry of polyhedral distortions. predictive relationships for the phosphate group. Acta Crystallogr. B30, 1195-1215 (1974).

8. Robinson, K., Gibbs, G. V. \& Ribbe, P. H. Quadratic elongation: a quantitative measure of distortion in coordination polyhedra. Science 172, 567-570 (1971).

9. Balic-Zunic, T. \& Makovicky, E. Determination of the centroid or 'the best centre' of a coordination polyhedron. Acta Crystallogr. B52, 78-81 (1996).

10. Balic-Zunic, T. \& Vickovic, I. IVTON-program for the calculation of geometrical aspects of crystal structures and some crystal chemical applications. J. Appl. Crystallogr 29, 305-306 (1996).

11. Collings, I. E. et al. Compositional dependence of anomalous thermal expansion in perovskite-like $\mathrm{ABX}_{3}$ formates. Dalton Trans. 45, 4169-4178 (2016).

12. Attfield, J. P., Lightfoot, P. \& Morris, R. E. Perovskites. Dalton Trans. 44, 10541-10542 (2015).

13. Glazer, A. M. The classification of tilted octahedra in perovskites. Acta Crystallogr. B28, 3384-3392 (1972).

14. Woodward, P. M. Octahedral tilting in perovskites. I. geometrical considerations. Acta Crystallogr. B53, 32-43 (1997).

15. Howard, C. J. \& Stokes, H. T. Group-theoretical analysis of octahedral tilting in perovskites. Acta Crystallogr. B54, 782-789 (1998).

16. Howard, C. J., Kennedy, B. J. \& Chakoumakos, B. C. Neutron powder diffraction study of rhombohedral rare-earth aluminates and the rhombohedral to cubic phase transition. J. Phys. Condens. Matter 12, 349-365 (2000).

17. Hayward, S. A. et al. Transformation processes in $\mathrm{LaAlO}_{3}$ : Neutron diffraction, dielectric, thermal, optical and Raman studies. Phys. Rev. B 72, 054110 (2005).

18. Megaw, H. D. \& Darlington, C. N. W. Geometrical and structural relations in the rhombohedral perovskites. Acta Crystallogr. A31, 161-173 (1975).

19. Devonshire, A. F. Theory of barium titanate - part I. Phil. Mag XCVI, 1040-1063 (1949).

20. Devonshire, A. F. Theory of barium titanate-part II. Phil. Mag. CIX, 1065-1079 (1951).

21. Comès, R., Lambert, M. \& Guinier, A. Désordre linéaire dans les cristaux (cas du silicium, du quartz, et des pérovskites ferroélectriques). Acta Crystallogr. A26, 244-254 (1970).

22. Senn, M. S., Keen, D. A., Lucas, T. C. A., Hriljac, J. A. \& Goodwin, A. L. Emergence of long-range order in $\mathrm{BaTiO}_{3}$ from local symmetry-breaking distortions. Phys. Rev. Lett. 116, 207602 (2016).

23. Kwei, G. H., Lawson, A. C. \& Billinge, S. J. L. Structures of the ferroelectric phases of barium titanate. J. Phys. Chem. 97, 2368-2377 (1993).

24. Miyake, S. \& Ueda, R. On phase transformation of $\mathrm{BaTiO}_{3}$. J. Phys. Soc. Jpn 2, 93-97 (1947).

25. Belsky, A., Hellenbrandt, M., Karen, V. L. \& Luksch, P. New developments in the Inorganic Crystal Structure Database (ICSD): accessibility in support of materials research and design. Acta Crystallogr. B58, 364-369 (2002).

26. Allen, F. H. The Cambridge Structural Database: a quarter of a million crystal structures and rising. Acta Crystallogr. 58, 380-388 (2002).

27. Iizumi, M. et al. Structure of magnetite $\left(\mathrm{Fe}_{3} \mathrm{O}_{4}\right)$ below the Verwey transition temperature. Acta Crystallogr. B38, 2121-2133 (1982).

28. Wright, J. P., Attfield, J. P. \& Radaelli, P. G. Charge ordered structure of magnetite $\mathrm{Fe}_{3} \mathrm{O}_{4}$ below the Verwey transition. Phys. Rev. B 66, 214422 (2002).

29. Walz, F. The Verwey transition-a topical review. J. Phys. Condens. Matter 14, R285-R340 (2002).
30. Goff, R. J., Wright, J. P., Attfield, J. P. \& Radaelli, P. G. Resonant x-ray diffraction study of the charge ordering in magnetite. J. Phys. Condens. Matter 17, 7633-7642 (2005).

31. Joly, Y. et al. Low-temperature structure of magnetite studied using resonant x-ray scattering. Phys. Rev. B 78, 134110 (2008).

32. Blasco, J., Garcia, J. \& Subias, G. Structural transformation in magnetite below the Verwey transition. Phys. Rev. B 83, 104105 (2011).

33. Senn, M. S., Wright, J. P. \& Attfield, J. P. Charge order and three-site distortions in the Verwey structure of magnetite. Nature 481, 173-176 (2012).

34. Attfield, J. P. The Verwey phase of magnetite: a long-running mystery in ferrites. J. Jpn Soc. Powder Powder Metall. 61, S43-S48 (2014).

35. Senn, M. S., Wright, J. P., Cumby, J. \& Attfield, J. P. Charge localization in the Verwey structure of magnetite. Phys. Rev. B 92, 024104 (2015).

36. Ok, K. M. et al. Distortions in octahedrally coordinated $\mathrm{d}^{0}$ transition metal oxides: a continuous symmetry measures approach. Chem. Mater. 18, 3176-3183 (2006).

37. Shen, S.-P. et al. Magnetic-ion-induced displacive electric polarization in $\mathrm{FeO}_{5}$ bipyramidal units of (Ba, Sr) $\mathrm{Fe}_{12} \mathrm{O}_{19}$ hexaferrites. Phys. Rev. B 90, 180404(R) (2014)

38. Todd, M. J. \& Yildirim, E. A. On Khachiyan's algorithm for the computation of minimum-volume enclosing ellipsoids. Discrete Appl. Math. 155, 1731-1744 (2007).

\section{Acknowledgements}

This work was supported by ERC Advanced Grant EC 339312. We thank Dr C. Lithgow for testing of the software.

\section{Author contributions}

J.C. and J.P.A. conceived and coordinated the study and wrote the paper.

J.C. implemented the algorithm and performed the ellipsoidal analyses.

\section{Additional information}

Supplementary Information accompanies this paper at http://www.nature.com/ naturecommunications

Competing financial interests: The authors declare no competing financial interests.

Reprints and permission information is available online at http://npg.nature.com/ reprintsandpermissions/

How to cite this article: Cumby, J. \& Attfield, J. P. Ellipsoidal analysis of coordination polyhedra. Nat. Commun. 8, 14235 doi: 10.1038/ncomms14235 (2017).

Publisher's note: Springer Nature remains neutral with regard to jurisdictional claims in published maps and institutional affiliations.

This work is licensed under a Creative Commons Attribution 4.0 International License. The images or other third party material in this article are included in the article's Creative Commons license, unless indicated otherwise in the credit line; if the material is not included under the Creative Commons license, users will need to obtain permission from the license holder to reproduce the material To view a copy of this license, visit http://creativecommons.org/licenses/by/4.0/

(C) The Author(s) 2017 